\title{
Usability Analysis of Collision Avoidance System in Vehicle-to-Vehicle Communication Environment
}

\author{
Hong Cho, Gyoung-Eun Kim, and Byeong-Woo Kim \\ Department of Electrical Engineering, University of Ulsan, No. 93, Daehak-ro, Nam-gu, Ulsan, Republic of Korea
}

Correspondence should be addressed to Byeong-Woo Kim; bywokim@ulsan.ac.kr

Received 31 January 2014; Accepted 4 March 2014; Published 10 April 2014

Academic Editor: Young-Sik Jeong

Copyright (C) 2014 Hong Cho et al. This is an open access article distributed under the Creative Commons Attribution License, which permits unrestricted use, distribution, and reproduction in any medium, provided the original work is properly cited.

Conventional intelligent vehicles have performance limitations owing to the short road and obstacle detection range of the installed sensors. In this study, to overcome this limitation, we tested the usability of a new conceptual autonomous emergency braking (AEB) system that employs vehicle-to-vehicle (V2V) communication technology in the existing AEB system. To this end, a radar sensor and a driving and communication environment constituting the AEB system were simulated; the simulation was then linked by applying vehicle dynamics and control logic. The simulation results show that the collision avoidance relaxation rate of V2V communication-based AEB system was reduced compared with that of existing vehicle-mounted-sensor-based system. Thus, a method that can lower the collision risk of the existing AEB system, which uses only a sensor cluster installed on the vehicle, is realized.

\section{Introduction}

In recent years, with the strict enforcement of safety regulations for vehicles, consumer interest in vehicle safety is growing. Therefore, studies on active safety systems and advanced driver assistance systems (ADASs) have been actively taken up with the aim of ensuring safety, including vehicle control for accident avoidance and mitigation; these features are in contrast with those of conventional passive systems, which ensure safety through simple warnings [1]. One such prominent active system is the autonomous emergency braking (AEB) system. In a recent survey, the European Union (EU) determined that introducing the AEB system could reduce the annual number of deaths and serious injuries in vehicle accidents by more than 8,000 and 20,000, respectively [2]. Generally, an AEB system employs environment-recognition sensors such as radar, lidar, and cameras for detecting risk factors $[3,4]$. However, the existing sensor-based systems are able to detect only those vehicles that are within the employed sensors' measurement ranges, and blind spots may occur owing to obstacles. In addition, under bad weather conditions, detection becomes impossible or the detection accuracy drops significantly. For overcoming the limitations of sensor-based systems, recently, with the advancement of IT technology, cooperative safety system has been introduced. This system is grafted with vehicle safety communication schemes such as vehicle-to-vehicle (V2V) communication and vehicle-to-infra (V2I) communication [5].

Currently, international standards for AEB systems are being formulated worldwide, and various studies on AEB systems are being conducted. The existing studies on AEB systems were conducted based on the performance of sensors employed in vehicles and, therefore, they have limitations concerning detection area [6]. For overcoming these limitations, the current study was conducted based on the cooperative safety system grafted with V2I communication [7]. A limitation of the AEB system, that is, the blind zone occurring at a crossroad, was partially solved through V2I communication by employing radars in traffic lights at crossroads. However, compared with direct V2V communication, V2I communication suffers from real-time limitations and limited detection areas of the sensors installed on the road surface. In the case of $\mathrm{V} 2 \mathrm{~V}$ communication, studies on the operating environment and evaluations of pure communication technologies were conducted using the existing sensorbased ADAS, and basic studies on cooperative adaptive cruise 


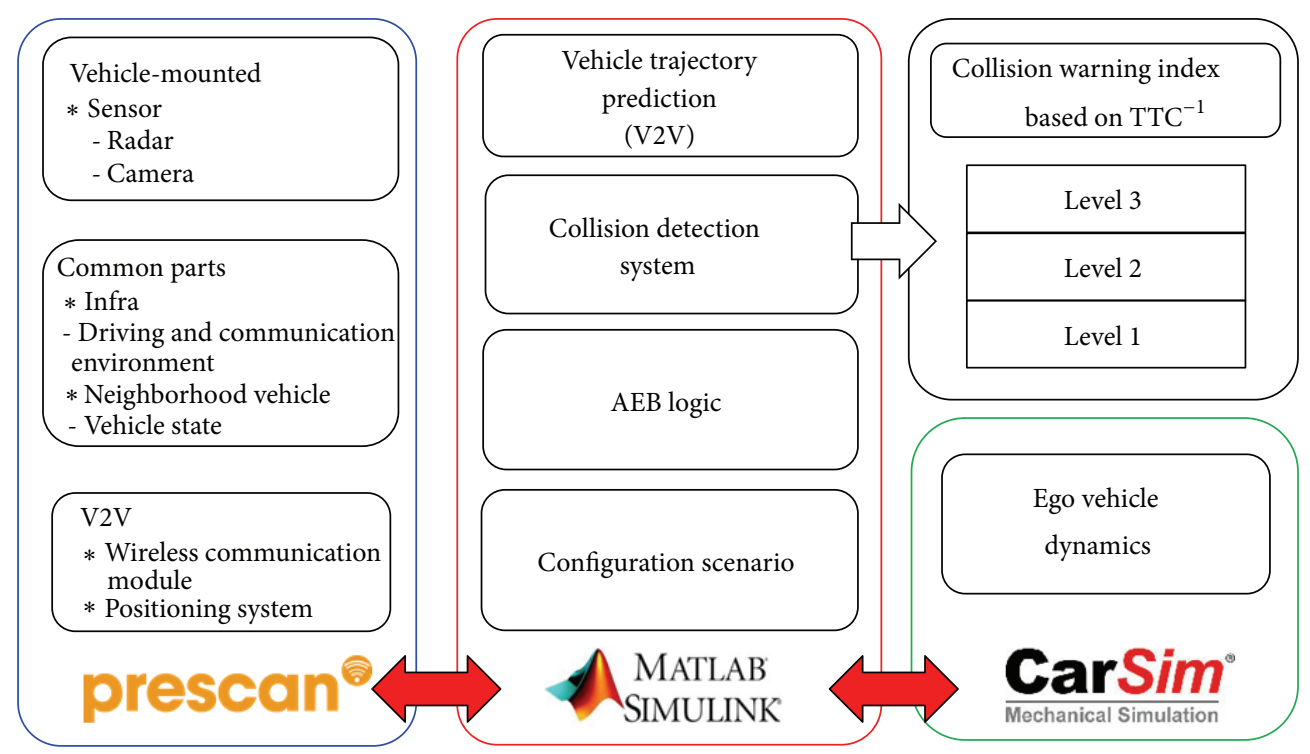

FIGURE 1: Block diagram of analysis model.

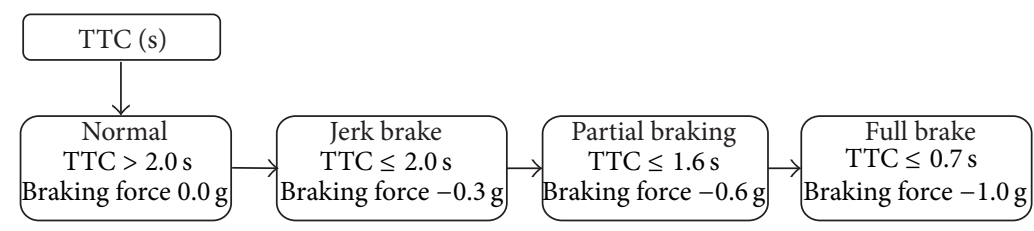

Figure 2: AEB logic.

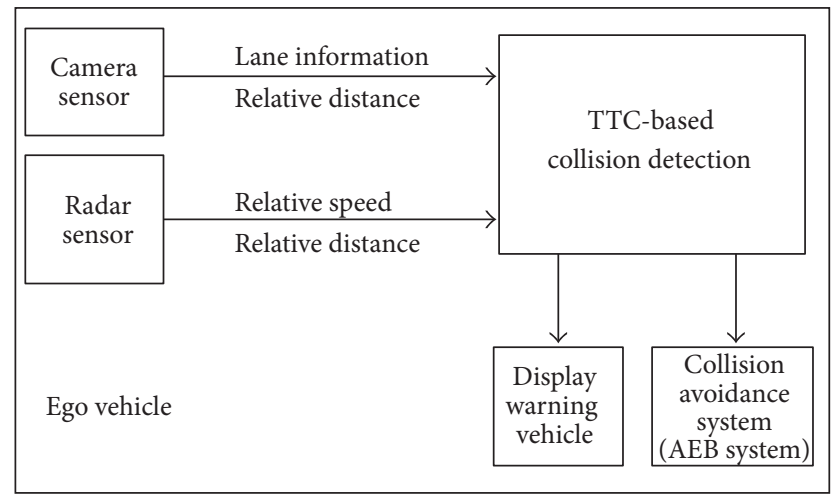

FIGURE 3: Block diagram of vehicle-mounted-sensor-based AEB system.

control were conducted by grafting ADAS with adaptive cruise control and realizing intervehicle communication [811]. Consequently, a presentation on the usability of the new conceptual AEB system that employs V2V communication was required.

Therefore, in this study, to overcome the aforementioned limitations of vehicle-mounted-sensor-based systems, we propose a new conceptual AEB system that employs $\mathrm{V} 2 \mathrm{~V}$ communication along with environment recognition sensors. In addition, the usability of V2V communication was compared with that of vehicle-mounted sensors by modifying an existing vehicle-mounted-sensor-based AEB system to incorporate $\mathrm{V} 2 \mathrm{~V}$ communication.

\section{AEB System Design}

2.1. AEB System Analysis Model. As shown in Figure 1, for analyzing the usability of $\mathrm{V} 2 \mathrm{~V}$ communication in comparison with that of vehicle-mounted sensors, a detailed model including various sensors and modules, the driving and communication environment, and vehicle dynamic characteristics was required. Therefore, in this study, PreScan, a commercial simulation code, was used for modeling wireless communication modules, high-precision location measurement systems, the driving and communication environment, and the radar and camera sensors installed in a vehicle. In addition, to determine the dynamic characteristics of a user vehicle equipped with the AEB system, a full car model with multiple degrees of freedom was generated in CarSim, a vehicle dynamic behavior simulation software application, and used. Finally, after interfacing PreScan and CarSim through MATLAB/Simulink, an analysis model was built based on a collision detection system, AEB logic, and the setting of a given scenario. 


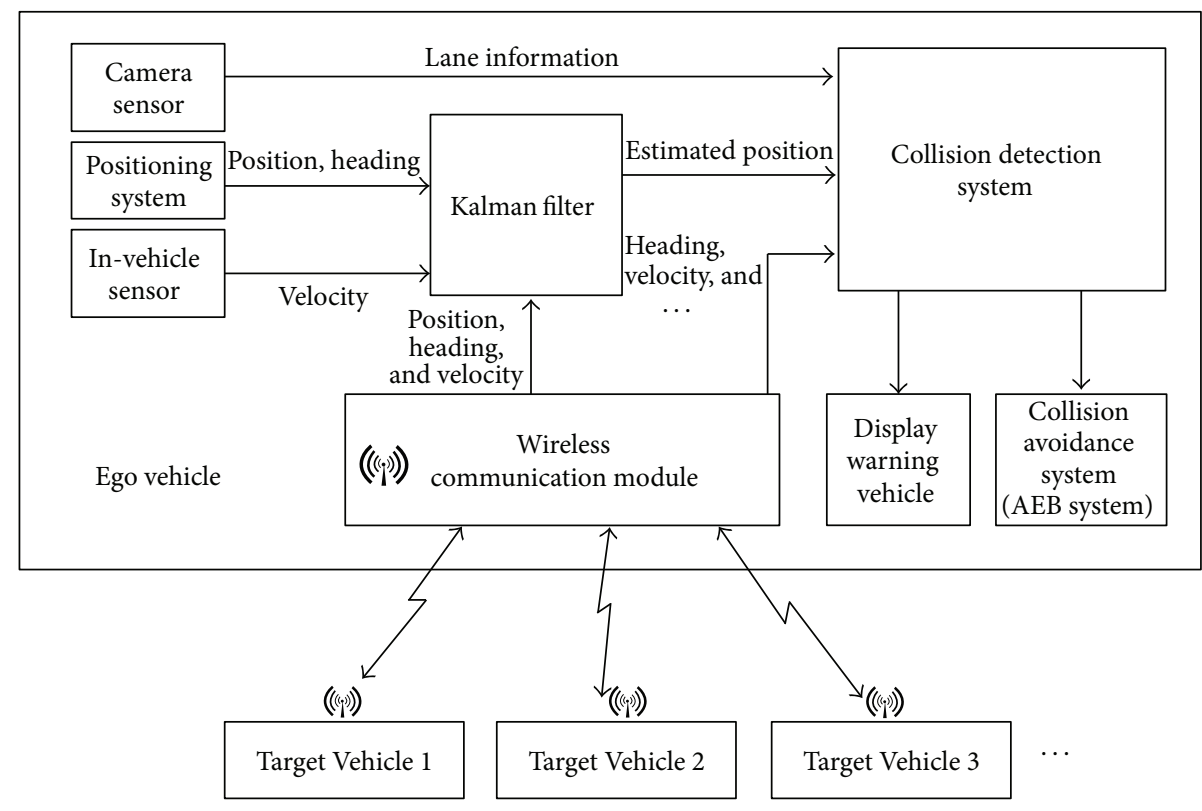

FIGURE 4: Block diagram of V2V communication-based AEB system.

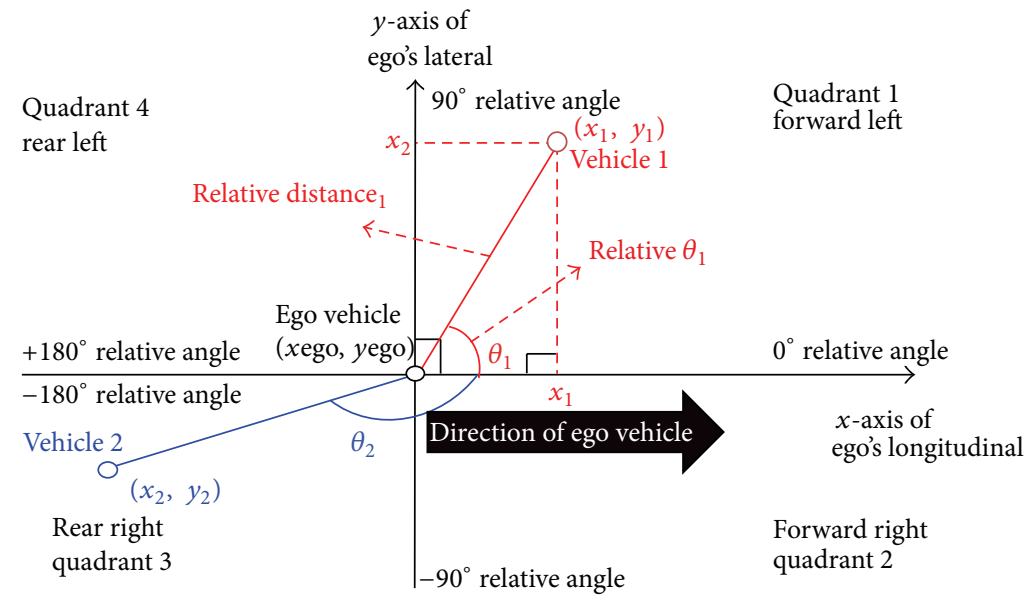

FIGURE 5: Principle of collision detection system.

2.2. AEB System. AEB is an active safety system that measures the degree of risk between a user vehicle and a forward vehicle using vehicle-installed environment recognition sensors such as radars or cameras. It helps in preventing accidents through automatic brake control in risky situations. In Europe, the enforcement of rules concerning AEB systems from 2014 has been initiated. In Europe, the rules concerning AEB systems have come into effect since the beginning of 2014. In 2009, it was proposed that an informal group, called the autonomous emergency braking system (AEBS)/lane departure warning system (LDWS) informal group, will be formed under the Working Party on Brakes and Running Gear (GRRF), a subsidiary body of a World Forum for Harmonization of Vehicle (WP.29), in order to formulate AEBS/LDWS standards. Economic Commission for Europe (ECE) regulations concerning AEBS are being enacted under the United Nations Economic Commission for Europe (UNECE) [12].
TABLE 1: AEB system braking force.

\begin{tabular}{lc}
\hline TTC $(\mathrm{s})$ & Braking force $(\mathrm{g})$ \\
\hline$\leq 2.0$ & 0.3 \\
$\leq 1.6$ & 0.6 \\
$\leq 0.7$ & 1.0 \\
\hline
\end{tabular}

The AEB system described in this paper meets the required performance specifications defined by the AEB Group. The time of application of automatic braking force by the AEB system was determined based on a collision risk index, that is, time-to-collision (TTC) of the user vehicle and a forward vehicle. TTC can be calculated using (1), which is based on the relative speed and relative distance between the user vehicle and forward vehicle. Table 1 and Figure 2 list and show, respectively, the braking force of the AEB system 


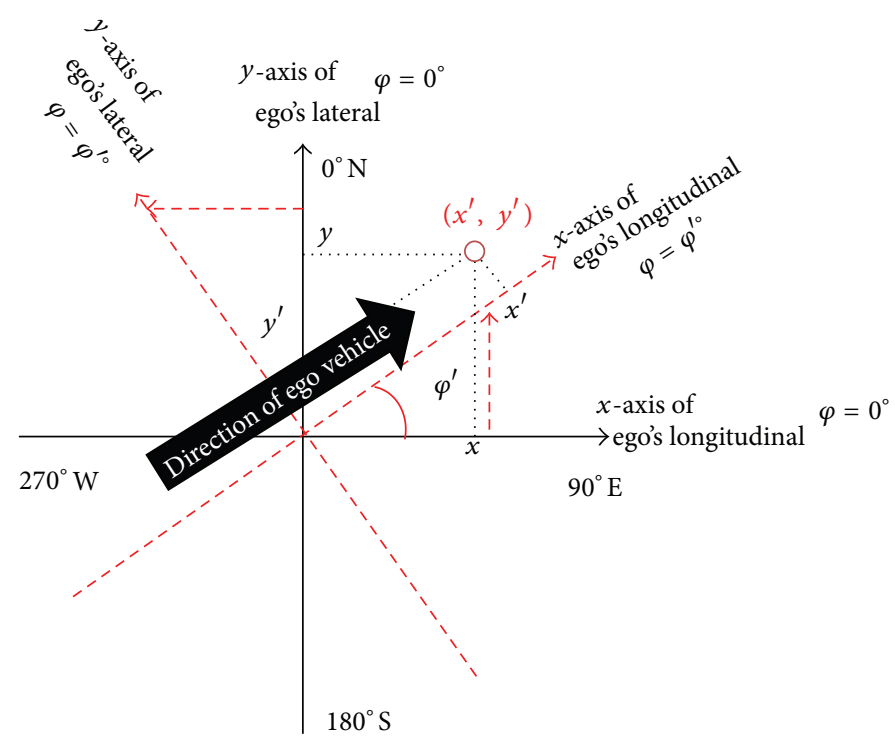

FIGURE 6: CSego transformation with azimuth change.

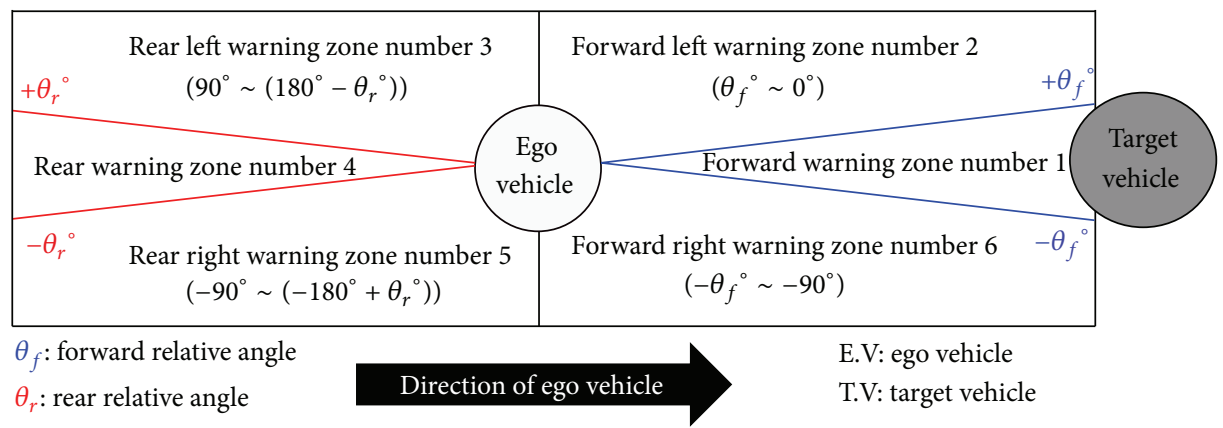

FIGURE 7: Warning zone corresponding to relative angle.

and AEB logic according to changes in TTC; here, $g$ denotes acceleration due to gravity and it is taken as $9.8 \mathrm{~m} / \mathrm{s}^{2}$ [7]. Consider

$$
\mathrm{TTC}(\mathrm{s})=\frac{\text { Relative distance }}{\text { Relative speed }}
$$

2.3. Vehicle-Mounted-Sensor-Based AEB System. Generally, a vehicle-mounted-sensor-based AEB system comprises a camera sensor and long- and short-distance radar sensors. Sensor specifications were determined by referring to the specifications of an actual commercial product. Table 2 lists the specifications of each sensor. The camera sensor mounted in the front provides information about the traffic in a lane and the relative distance to a forward obstacle. Long- and short-distance radar sensors can be used for measuring the distance and speed relative to an obstacle within the forward detection area. In this study, to compensate for the limitations of the camera and radar sensors, the distance and speed relative to a forward obstacle were measured through sensor fusion [13]. The TTC was calculated using the measured information and used as the brake input reference for the AEB system. Figure 3 shows a block diagram of a vehiclemounted-sensor-based AEB system.

2.4. V2V Communication-Based AEB System. The V2V communication-based AEB system described in this paper was operated based on the collision detection system proposed herein. During operation, the system received information about the nearby vehicles and the user vehicle through V2V communication and employed it for operation. Figure 4 shows the overall system configuration.

First, the location measuring system provides the vehicle location and heading angle information; this system included a noise model corresponding to the tolerance specification (Table 3) of commercial differential global positioning systems (GPS). The received GPS coordinates of the user vehicle and nearby vehicles were expressed in the $x, y$ coordinate system. The $x, y$ coordinate system defines a tangent plane in the GPS coordinates of the Infrasystem within $1 \mathrm{~km}$; the $x$-axis was defined toward the east and the $y$-axis toward the north [14]. Next, various sensors employed in the user vehicle transmit various types of information such as speed, acceleration, and yaw rate through the vehicle's internal 
TABLE 2: Vehicle-mounted sensors' specifications.

\begin{tabular}{lcc}
\hline & & Parameter \\
\hline Camera sensor & Focal length $(\mathrm{mm})$ & 6 \\
& FoV $\left(^{\circ}\right)$ & Azimuth: \pm 20.5 \\
& Elevation: \pm 13.5 & $752 \times 480$ \\
\hline Short-range radar & Resolution (pixels) & $0.2-30$ \\
& Detection range (m) & Azimuth: \pm 40 \\
& FoV $\left(^{\circ}\right)$ & Elevation: \pm 15 \\
\hline Long-range radar & Detection range (m) & $2-200$ \\
& FoV $\left(^{\circ}\right)$ & Azimuth: \pm 10 \\
& & Elevation: \pm 2.25 \\
\hline
\end{tabular}

TABLE 3: Module and sensor specifications of V2V communication-based AEB system.

\begin{tabular}{lcc}
\hline & & Parameter \\
\hline Camera sensor & Focal length (mm) & 6 \\
& FoV $\left(^{\circ}\right)$ & Azimuth: \pm 20.5 \\
Elevation: \pm 13.5 & $752 \times 480$ \\
\hline Position system (DGPS) & Resolution (pixels) & 50 \\
\hline Wireless communication module (V2V) & Accuracy (cm) & 1,000 \\
& Communication range (m) & Basic safety \\
& Message frame & Message (BSM) \\
\hline
\end{tabular}

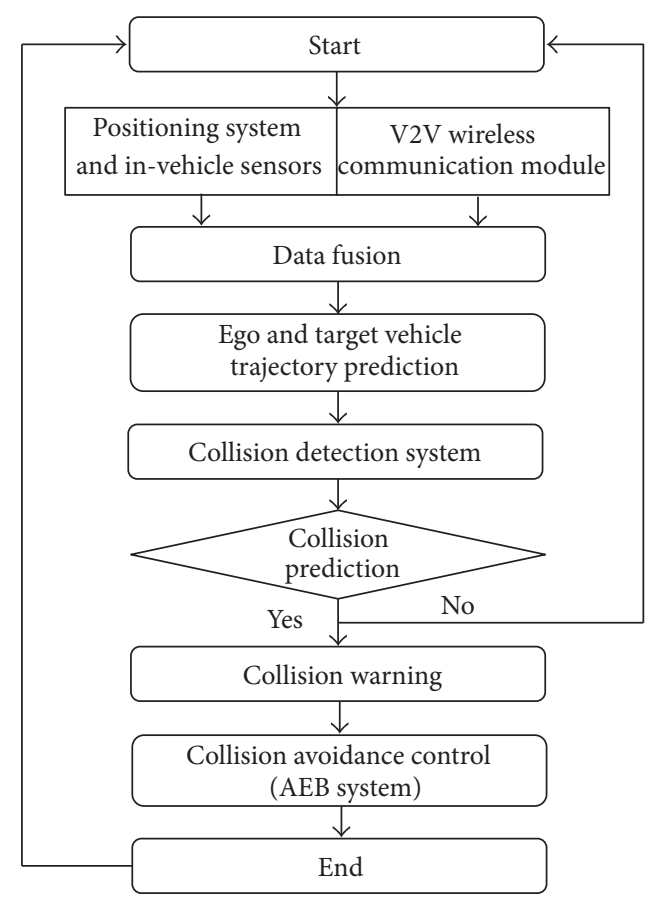

FIGURE 8: Flowchart of V2V communication-based AEB system.

communication system. Finally, the wireless communication module employed for intervehicle communication provides information about the nearby vehicles; a message frame received through the intervehicle communication channel was applied in conjunction with the basic safety message

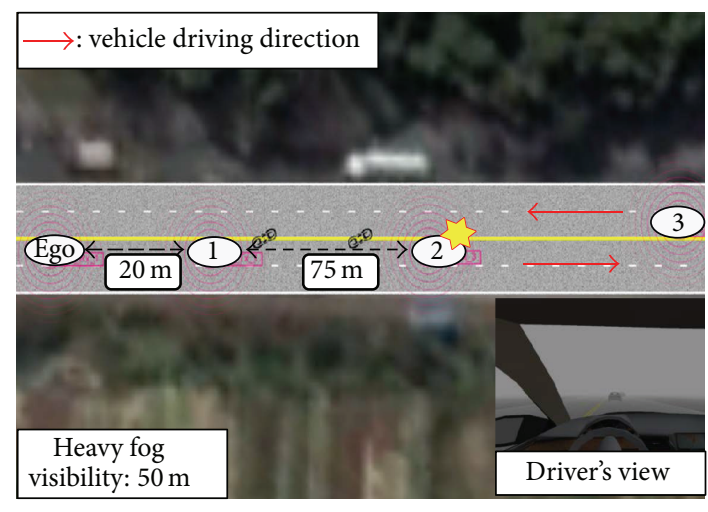

FIGURE 9: Initial road condition.

(BSM) standards defined in SAE J2735 [15]. In addition, error of the location measurement systems in the user vehicle and nearby vehicles was calibrated by employing a Kalman Filter; trajectories of the user's and nearby vehicles were measured $[16,17]$.

As shown in Figure 5, the V2V communication-based collision detection system proposed in this study determines the location of and distance to a nearby vehicle after generating CSego, a Cartesian coordinate system, with the current location (xego, yego) of the user vehicle as its origin. CSego expresses the longitudinal direction along the $x$-axis and the transverse direction along the $y$-axis with reference to the user vehicle's driving direction. The locations of nearby vehicles in CSego are divided along the quadrants of CSego and expressed in relative coordinates $(x n, y n)(n=$ vehicle id) 


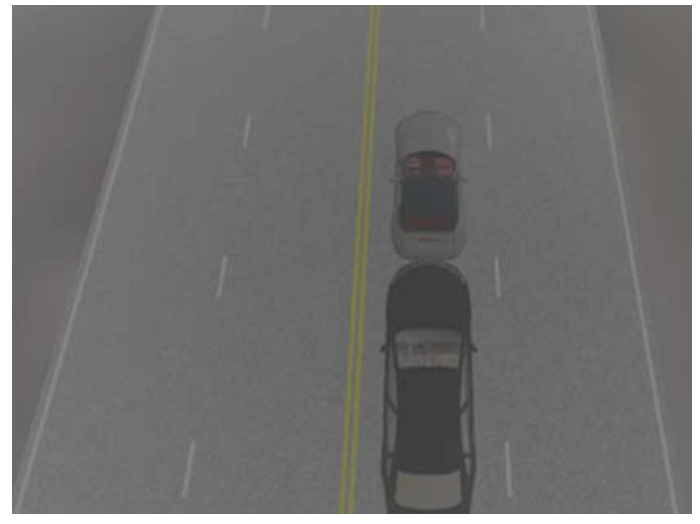

(a) Simulation viewer

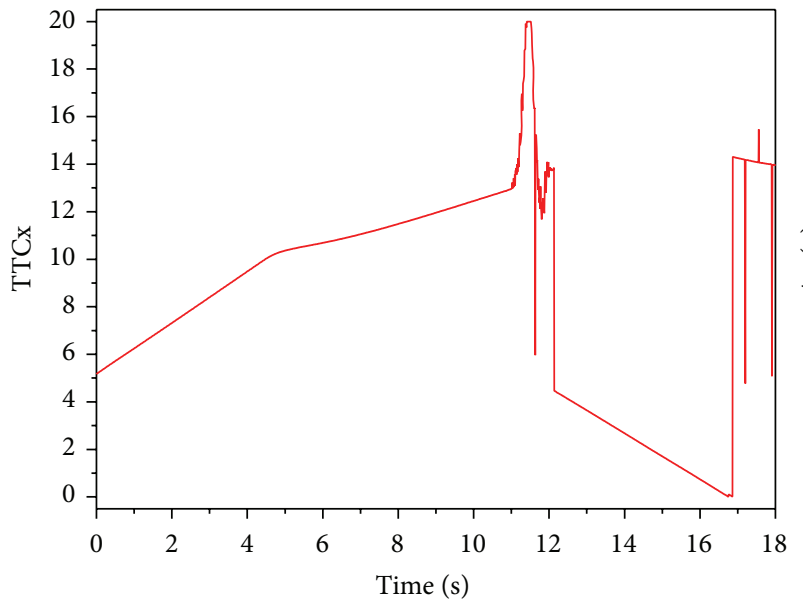

(c) TTCx

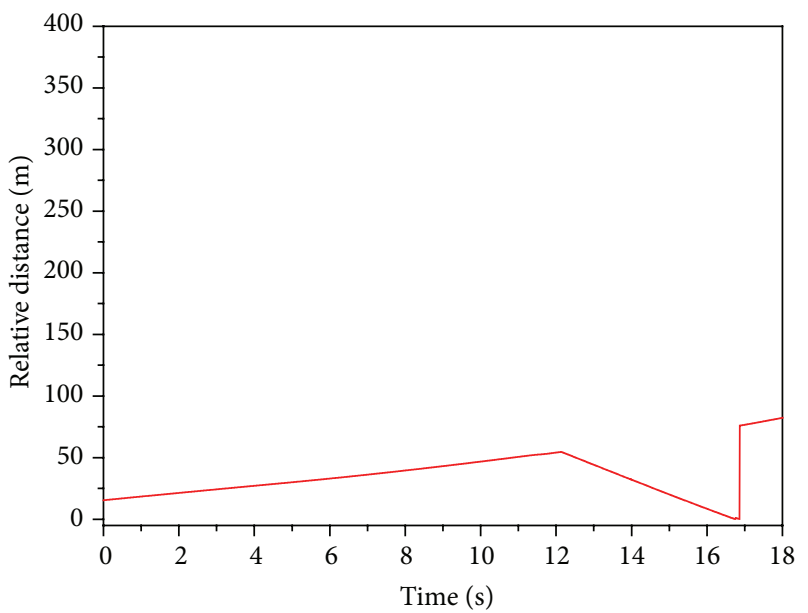

(e) Relative distance

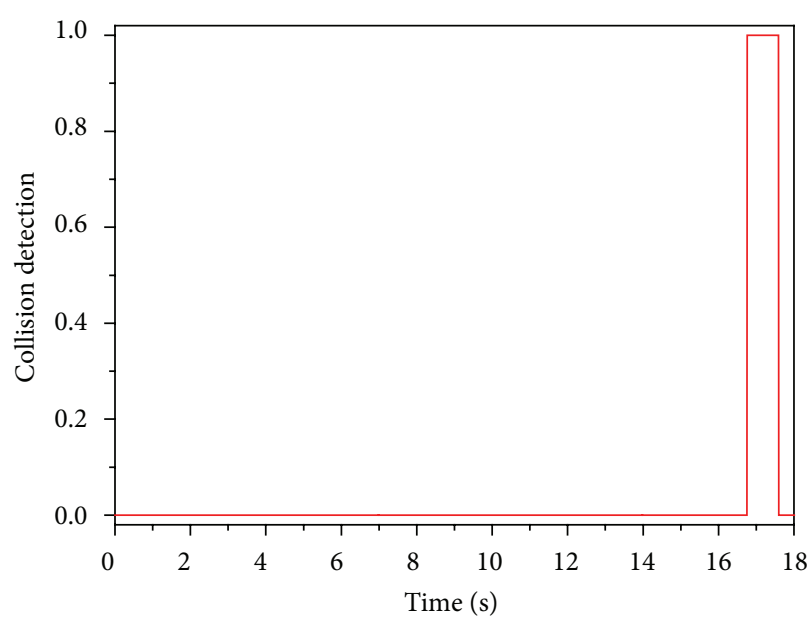

(b) Collision detection flag

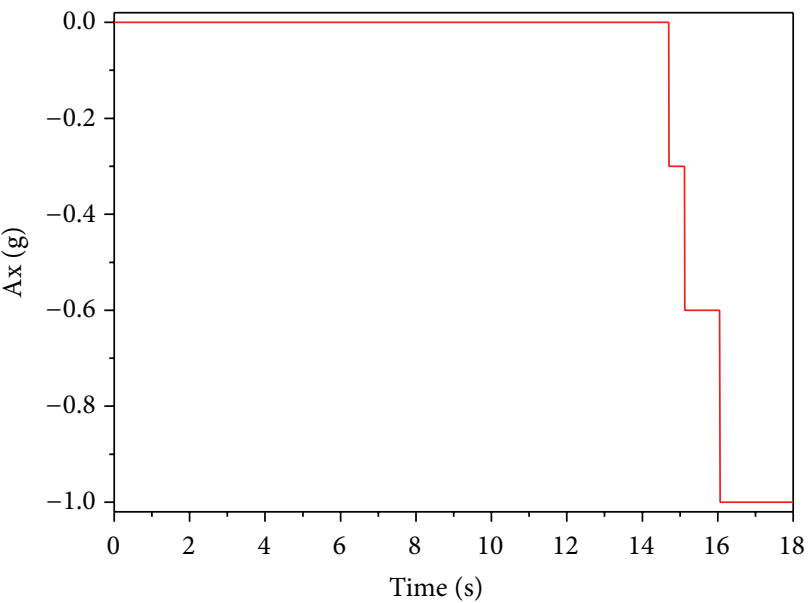

(d) AEB system command

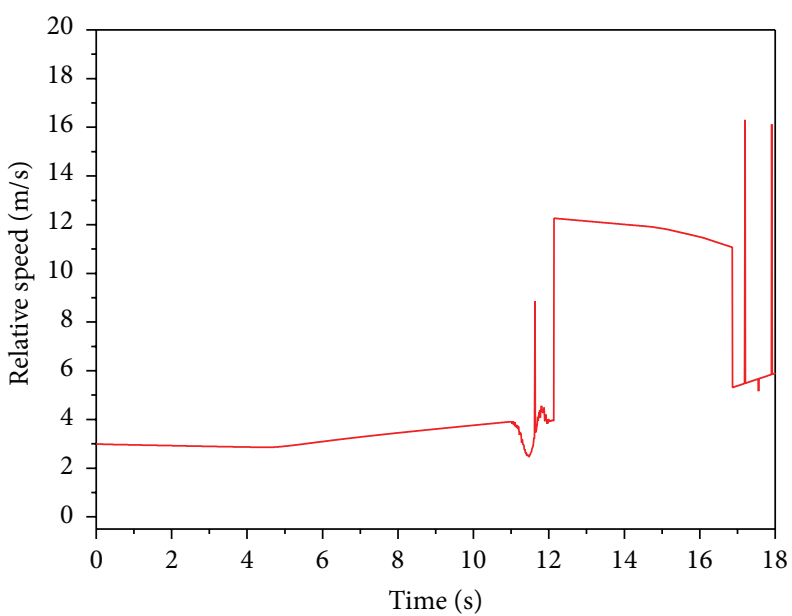

(f) Relative speed

Figure 10: Vehicle-mounted-sensor-based AEB system. 
after comparing the current location information of the user vehicle and nearby vehicles received through intervehicle communication. The relative angle $\theta n$ was calculated by comparison with the azimuth $\varphi$, which represents the user vehicle's driving direction. As shown in Figure 5, the nearby vehicle observation system can recognize the locations of nearby vehicles based on the relative angle $\theta n$, which varies along the quadrant. As shown in Figure 6, $\varphi$ varied with the user vehicle's heading angle and was set to be $0^{\circ}$ with respect to the east direction.

As shown in Figure 6, the coordinate axis of CSego rotated according to changes in $\varphi$. The longitudinal driving direction of the user vehicle was always matched with the $x$ axis using the rotational transformation matrix equation (2), which considers the coordinate axis' rotation

$$
\left[\begin{array}{l}
x^{\prime} \\
y^{\prime}
\end{array}\right]=\left[\begin{array}{cc}
\cos \varphi & \sin \varphi \\
-\sin \varphi & \cos \varphi
\end{array}\right]\left[\begin{array}{l}
x \\
y
\end{array}\right] .
$$

As shown in Figure 7, the warning zone was determined based on the relative angle obtained following the above process. $\theta f$ and $\theta r$ are the error ranges of the relative angle in the case that the user vehicle and all vehicles in the same traffic lane move in the middle of the traffic lane.

The relative angle, relative distance, and heading angle of the nearby vehicle are the parameters that determine the warning zone. The heading angle is an important parameter for determining the nearby vehicle's driving direction.

The relative heading angle between the user vehicle and the nearby vehicle was obtained for determining whether the nearby vehicle is driving in the same direction as the user vehicle, entering a crossroad, or driving in the opposite traffic lane. In addition, the relative distance can be obtained using the user vehicle's barycentric coordinate system CSego. However, the relative distance obtained thus does not account for the size of the vehicle; therefore, the relative distance was calibrated assuming a circular vehicle [18]. The TTC was calculated based on the obtained relative distance and relative speed of the user vehicle and the nearby vehicle, and the calculated TTC was used as the collision risk index for the AEB system. Figure 8 shows a flowchart of the V2V communication-based AEB system.

\section{Simulation and Results}

3.1. Simulation Scenario. As shown in Figure 9, the driving direction and scenarios were defined for a comparative analysis of the vehicle-mounted-sensor-based and V2V communication-based AEB systems. The driving direction was determined based on the conditions under which accidents generally occur. This includes the condition of heavy fog, under which visibility is less than $50 \mathrm{~m}$, which makes it difficult for a driver to recognize forward risk situations.

An ego vehicle mounted with an AEB system can avoid and mitigate the effects of collisions in the longitudinal direction. Consider the following scenario. The driver of Vehicle 1 changes lanes to avoid collision after finding that Vehicle 2 in front is stationary. Vehicle 3 is driving in the opposite traffic lane. The simulation scenario is summarized in Table 4.

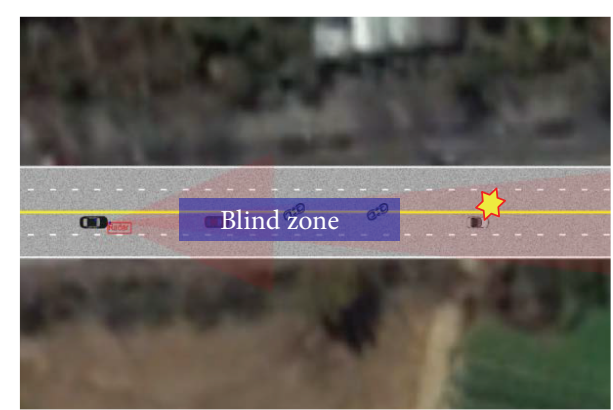

FIGURE 11: Limitations of vehicle-mounted sensor.

TABLE 4: Simulation scenario.

\begin{tabular}{lccc}
\hline Vehicle & Initial speed & End speed & Note \\
\hline Ego & $50 \mathrm{~km} / \mathrm{h}$ & $60 \mathrm{~km} / \mathrm{h}$ & AEB system \\
Vehicle 1 & $60 \mathrm{~km} / \mathrm{h}$ & $60 \mathrm{~km} / \mathrm{h}$ & Lane change \\
Vehicle 2 & $55 \mathrm{~km} / \mathrm{h}$ & $0 \mathrm{~km} / \mathrm{h}$ & Vehicle fault \\
Vehicle 3 & $70 \mathrm{~km} / \mathrm{h}$ & $70 \mathrm{~km} / \mathrm{h}$ & Opposite lane \\
\hline
\end{tabular}

3.2. Simulation Results. Simulation was performed by employing the vehicle-mounted-sensor-based AEB system and the $\mathrm{V} 2 \mathrm{~V}$ communication-based AEB system in the scenarios defined in Table 4.

Figure 10 shows the simulation results of the vehiclemounted-sensor-based AEB system. The system was capable of detecting forward vehicles only, such as the ego in this case, located in the traffic lane. Therefore, the longitudinal collision risk index, TTCx, shown in Figure 10(c), increased gradually from $0 \mathrm{~s}$ to about $11 \mathrm{~s}$ and then decreased rapidly. This is because Vehicle 1, which was running initially on the same traffic lane, changed lanes owing to the detection of a stationary vehicle; the sensor in the Ego vehicle detected a stationary vehicle (Vehicle 2) on the same traffic lane. As shown in Figure 10(d), it can be seen that the AEB system was applied normally with braking force as TTCx varied. However, it can be confirmed from the relative distance graph in Figure 10(e) that collision was predicted when the relative distance changed to $0 \mathrm{~m}$. In fact, even in the simulation environment, the occurrence of a collision can be confirmed based on the vehicle driving state shown in Figure 10(a) and the collision detection flag shown in Figure 10(b). In addition, a comparison of the relative speed before the time (about $14.8 \mathrm{~s}$ ) of braking force application by the AEB system, and the relative speed at the time of collision, shown in the relative speed graph of Figure 10(f), indicates that the speed was reduced by about $1.8 \mathrm{~km} / \mathrm{h}(0.5 \mathrm{~m} / \mathrm{s})$. Therefore, it can be said that the collision avoidance relaxation rate achieved with the vehicle-mounted-sensor-based AEB system in relevant scenarios was not more than $3 \%$. This can be ascribed to the vehicle-mounted sensors' inability to detect the stationary vehicle (Vehicle 2) on the road ahead owing to the presence of a blind zone due to a front vehicle, as shown in Figure 11.

In contrast, in the case of the $\mathrm{V} 2 \mathrm{~V}$ communication-based AEB system shown in Figures 12(a) and 12(b), there was no collision between the Ego vehicle and the stationary vehicle (Vehicle 2). The TTCx results shown in Figure 12(c) indicate 


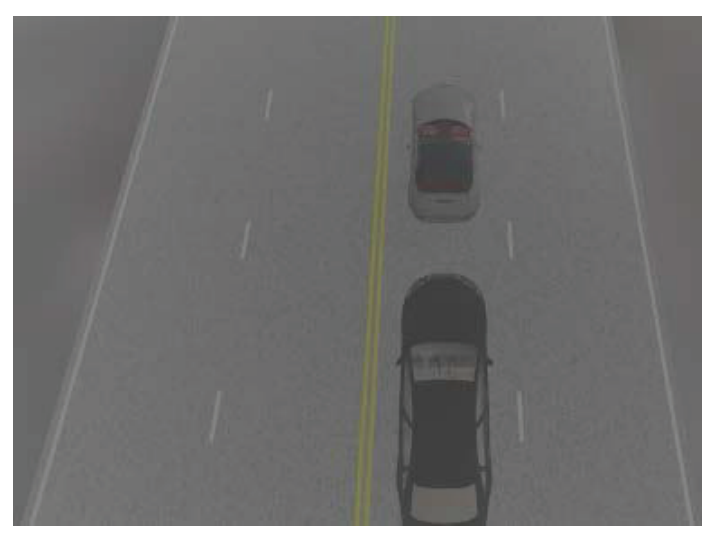

(a) Simulation viewer

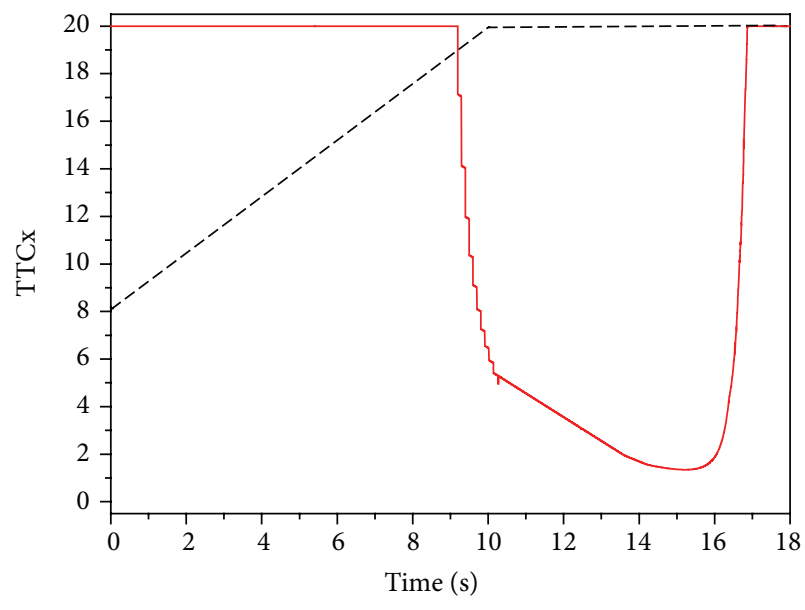

Vehicle 1

Vehicle 2

(c) TTCx

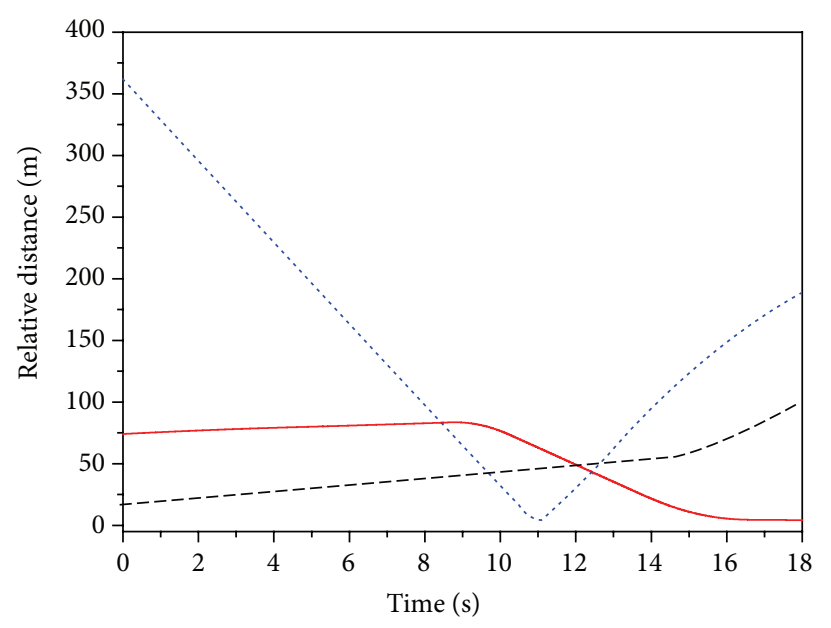

--- Vehicle $1 \quad$....... Vehicle 3

(e) Relative distance

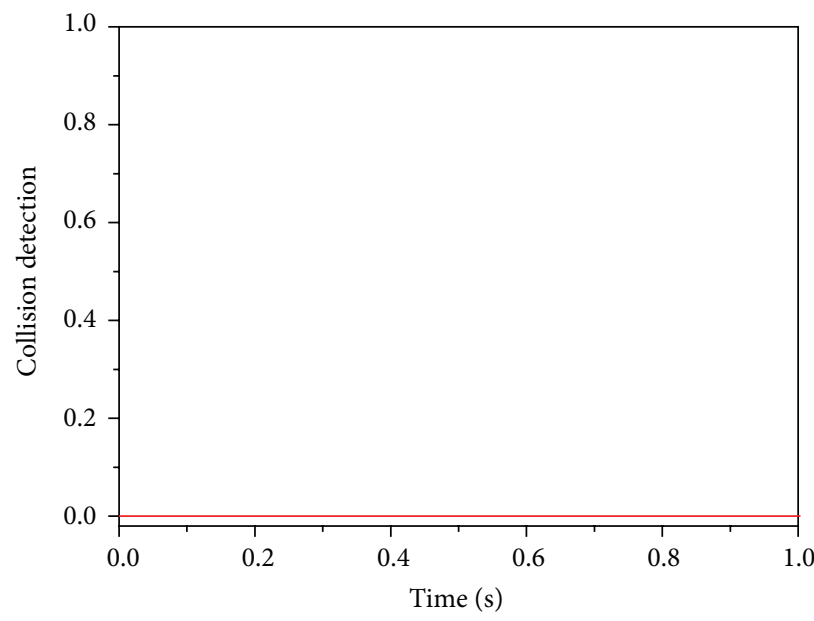

(b) Collision detection flag

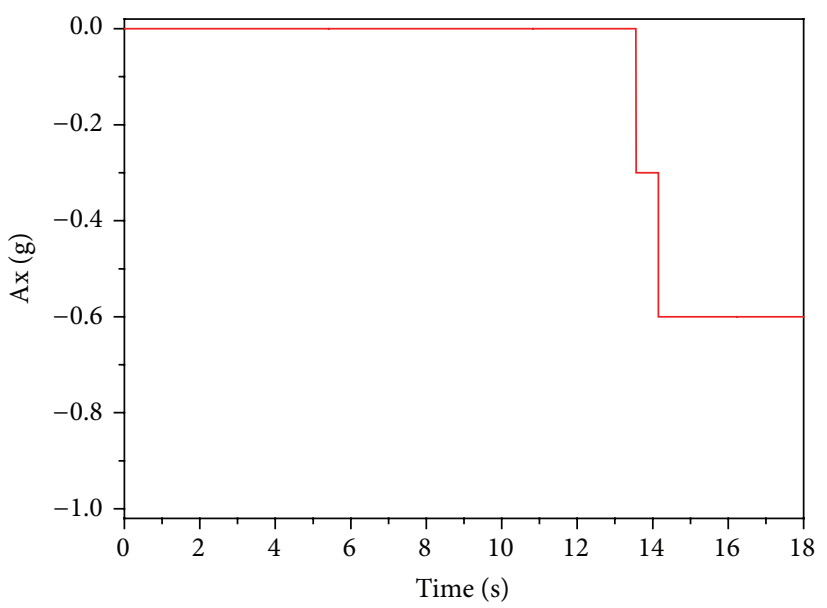

(d) AEB system command

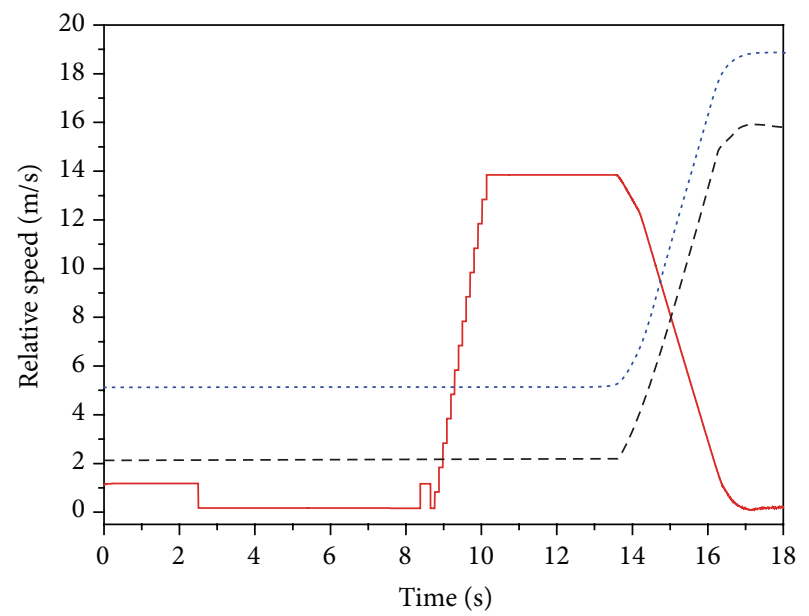

- - - Vehicle 1

Vehicle 3

(f) Relative speed 


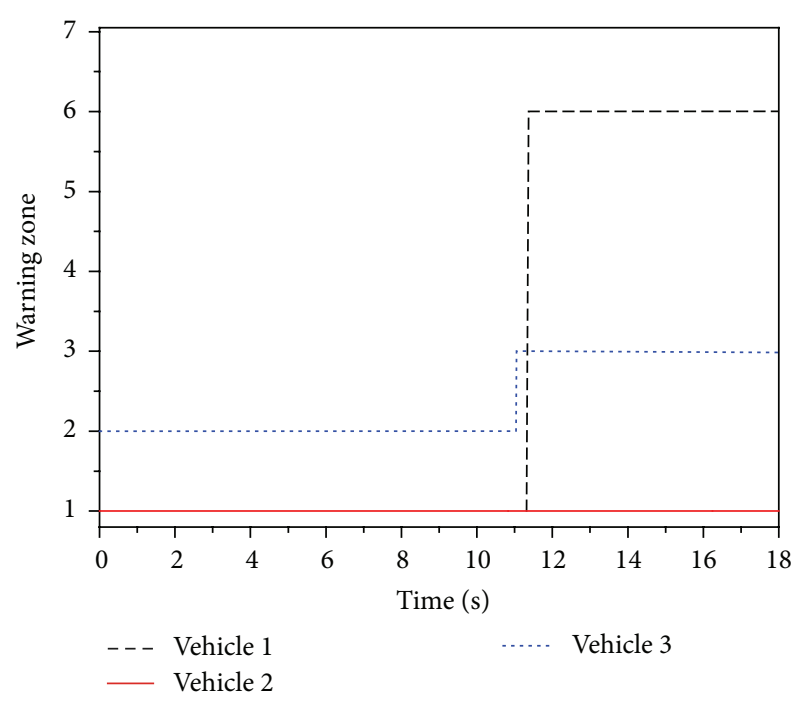

FIGURE 13: Warning zone change during simulation.

that there was a collision risk in the longitudinal direction with Vehicles 1 and 2, which were located in front of the Ego vehicle. However, Vehicle 3 was not represented in the TTCx graph. Vehicle 3 was determined to be a vehicle in the opposite traffic lane based on heading angle information received through $\mathrm{V} 2 \mathrm{~V}$ communication and was excluded by the collision detection system. It can be inferred from Figure 12(d) that braking force can be applied stably based on changes in the TTCx calculated by the collision detection system after predetecting the forward stationary vehicle (Vehicle 2) through intervehicle communication. In addition, the plots of relative distance (Figure 12(e)) and relative speed (Figure 12(f)) indicate that the collision avoidance relaxation rate reached $100 \%$ with the avoidance of collision because the relative speed decreased to $0 \mathrm{~m} / \mathrm{s}$ before the relative distance to Vehicle 2 decreased to $0 \mathrm{~m}$. In addition, it can be seen from Figure 12(f) that the relative speed with respect to Vehicle 2 increased slowly after about $9 \mathrm{~s}$, indicating that the vehicle became stationary at about $9 \mathrm{~s}$.

Figure 13 shows the warning zone according to simulation time. It can be seen that Vehiclel, which was running in front of the Ego vehicle, changed its traffic lane after detecting a stationary vehicle. Thus, the warning zone of Vehicle 1 was changed from 1 to 6 . Vehicle 2 was the stationary vehicle and there was no change in its traffic lane; thus, there was no change in its warning zone. Finally, Vehicle 3 was driving on the opposite traffic lane, and its warning zone changed from 2 to 3 at about $11.5 \mathrm{~s}$ because Vehicle 3 overtook the user vehicle. This can also be seen in the relative distance graph (Figure 12(e)), which shows that the relative distance with respect to Vehicle 3 was closer to 0 at about $11.5 \mathrm{~s}$ and started increasing thereafter.

\section{Conclusions}

In this study, the usability of the proposed V2V communication-based AEB system was compared with that of the existing vehicle-mounted-sensor-based system.
An analysis model was built for determining the usability of the V2V communication-based AEB system. The analysis model considered the vehicle-mounted sensor and V2V communication environments. Furthermore, the existing vehicle-mounted-sensor-based $A E B$ system was realized using this model. In addition, a new conceptual AEB system was proposed and developed by combining V2V communication technology with environment-recognition sensors. Then, a comparative analysis simulation of the $\mathrm{V} 2 \mathrm{~V}$ communication-based AEB system versus the vehiclemounted-sensor-based system was conducted in the same scenario. The simulation results show that in the case of the existing vehicle-mounted-sensor-based AEB system, braking application time lengthened and a collision occurred owing to the system's detection area limitation. However, in the case of the $\mathrm{V} 2 \mathrm{~V}$ communication-based AEB system, collision was avoided regardless of driving conditions and obstacles through collision risk detection within the range of intervehicle communication. In addition, in the case of the existing vehicle-mounted-sensor-based AEB system, the collision avoidance relaxation rate was no more than $3 \%$. In contrast, in the case of the V2V communication-based AEB system, the collision avoidance relaxation rate reached $100 \%$.

Therefore, the usability of the V2V communication technology was demonstrated through the aforementioned comparative analysis. Future studies will be aimed at testing the proposed system in the $\mathrm{V} 2 \mathrm{~V}$ communication environment with an actual vehicle used in practice and analyzing the proposed system in various scenarios and driving environments.

\section{Conflict of Interests}

The authors declare that there is no conflict of interests regarding the publication of this paper.

\section{Acknowledgments}

This research was supported by the Ministry of Science, ICT, and Future Planning (MSIP), Korea, under the Convergence Information Technology Research Center (C-ITRC) support program (NIPA-2013-H0401-13-1008) supervised by the National IT Industry Promotion Agency (NIPA). And this paper is an extended and improved version of the paper accepted for the KCIC-2013/FCC-2014 Conferences.

\section{References}

[1] M. Lu, K. Wevers, R. Van Der Heijden, and T. Heijer, "ADAS applications for improving traffic safety," in Proceedings of the IEEE International Conference on Systems, Man and Cybernetics (SMC '04), vol. 4, pp. 3995-4002, October 2004.

[2] Economic Commision for Europe, "An introduction to the new vehicle safety regulation,” Tech. Rep. WP.92-145-08, Informal Document, Geneva, Switzerland, 2008.

[3] K. Goswami, G. S. Hong, and B. G. Kim, "A novel mesh-based moving object detection technique in video sequence," Journal of Convergence, vol. 4, no. 3, pp. 20-24, 2013. 
[4] Verma, V. Jain, and R. Gumber, "Simple fuzzy rule-based edge detection," Journal of Information Processing Systems, vol. 9, no. 4, pp. 575-591, 2013.

[5] D. Caveney and W. B. Dunbar, "Cooperative driving: beyond $\mathrm{V} 2 \mathrm{~V}$ as an ADAS sensor," in Proceedings of the IEEE Intelligent Vehicles Symposium (IV '12), pp. 529-534, June 2012.

[6] N. Kaempchen, B. Schiele, and K. Dietmayer, "Situation assessment of an autonomous emergency brake for arbitrary vehicleto-vehicle collision scenarios," IEEE Transactions on Intelligent Transportation Systems, vol. 10, no. 4, pp. 678-687, 2009.

[7] J. Lee, S. Jo, J. Kwon, T. Hong, and K. Park, "Development of V2I-based Intersection Collision Avoidance System," in Proceedings of the Conference for Korea Institute of ITS, pp. 9096, May 2013.

[8] G. Peng, K. Zeng, and X. Yang, "A hybrid computational intelligence approach for the VRP problem," Journal of Convergence, vol. 4, no. 2, pp. 1-4, 2013.

[9] S. Wang, A. Huang, and T. Zhang, "Performance evaluation of IEEE 802.15.4 for V2V communication in VANET," in Proceedings of the International Conference on Computational and Information Sciences, pp. 1603-1606, June 2013.

[10] Z. Taysi and A. Yavuz, "ETSI compliant GeoNetworking protocol layer implementation for IVC simulations," Human-Centric Computing and Information Sciences, vol. 3, no. 1, p. 4, 2013.

[11] C. Desjardins and B. Chaib-Draa, "Cooperative adaptive cruise control: a reinforcement learning approach," IEEE Transactions on Intelligent Transportation Systems, vol. 12, no. 4, pp. 12481260, 2011.

[12] Economic Commission for Europe, Automatic Emergency Braking and Lane Departure Warning Systems, UN/ECE/ TRANS/WP29/GRRF-65-Inf19, Informal Group on Automatic Emergency Braking and Lane Departure Warning System, Geneva, Switzerland, 2009.

[13] J. S. Sangorrin, J. Sparbert, U. Ahlrichs, W. Branz, and O. Schwindt, "Sensor data fusion for active Safety Systems," SAE International Journal of Passenger Cars-Electronic and Electrical Systems, vol. 3, no. 2, pp. 154-161, 2010.

[14] “PreScan R6. 6. 0 Help Manual,” May 2013.

[15] C. Hedges and F. Perry, "Overview and use of SAE J2735 message sets for commercial vehicles," SAE Technical Paper 2008-01-2650, October 2008.

[16] J. Huang and H.-S. Tan, "A low-order DGPS-based vehicle positioning system under urban environment," IEEE/ASME Transactions on Mechatronics, vol. 11, no. 5, pp. 567-575, 2006.

[17] S. Ammoun, F. Nashashibi, and C. Laurgeau, "Real-time crash avoidance system on crossroads based on 802.11 devices and GPS receivers," in Proceedings of the IEEE Intelligent Transportation Systems Conference (ITSC '06), pp. 1023-1028, September 2006.

[18] Y. Wang, "Vehicle collision warning system and collision detection algorithm based on vehicle infrastructure integration," in Proceedings of the 7th Advanced Forum on Transportation of China (AFTC '11), pp. 216-220, October 2011. 


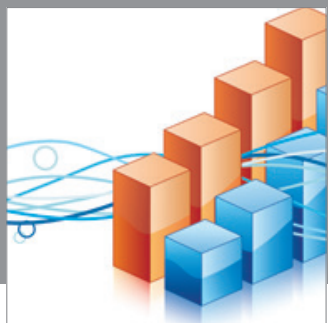

Advances in

Operations Research

mansans

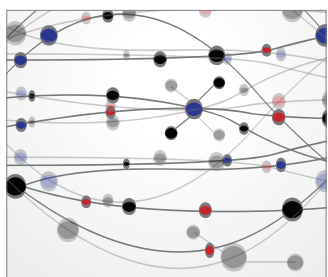

The Scientific World Journal
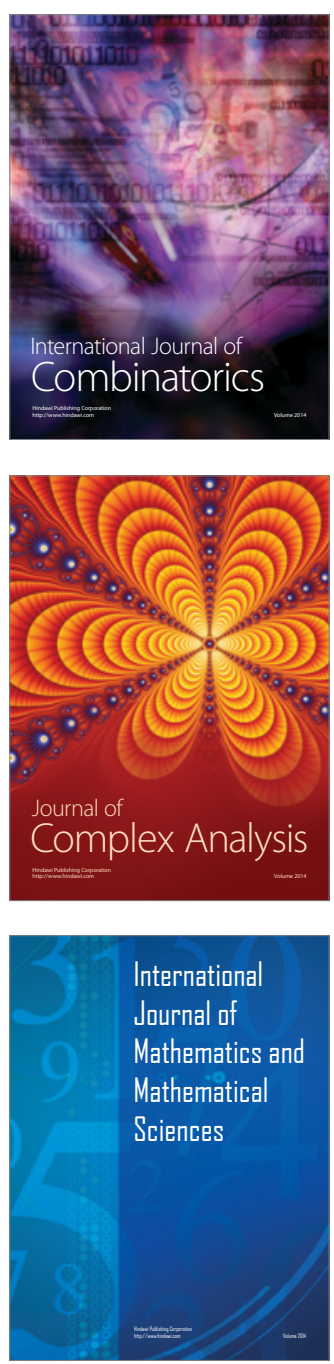
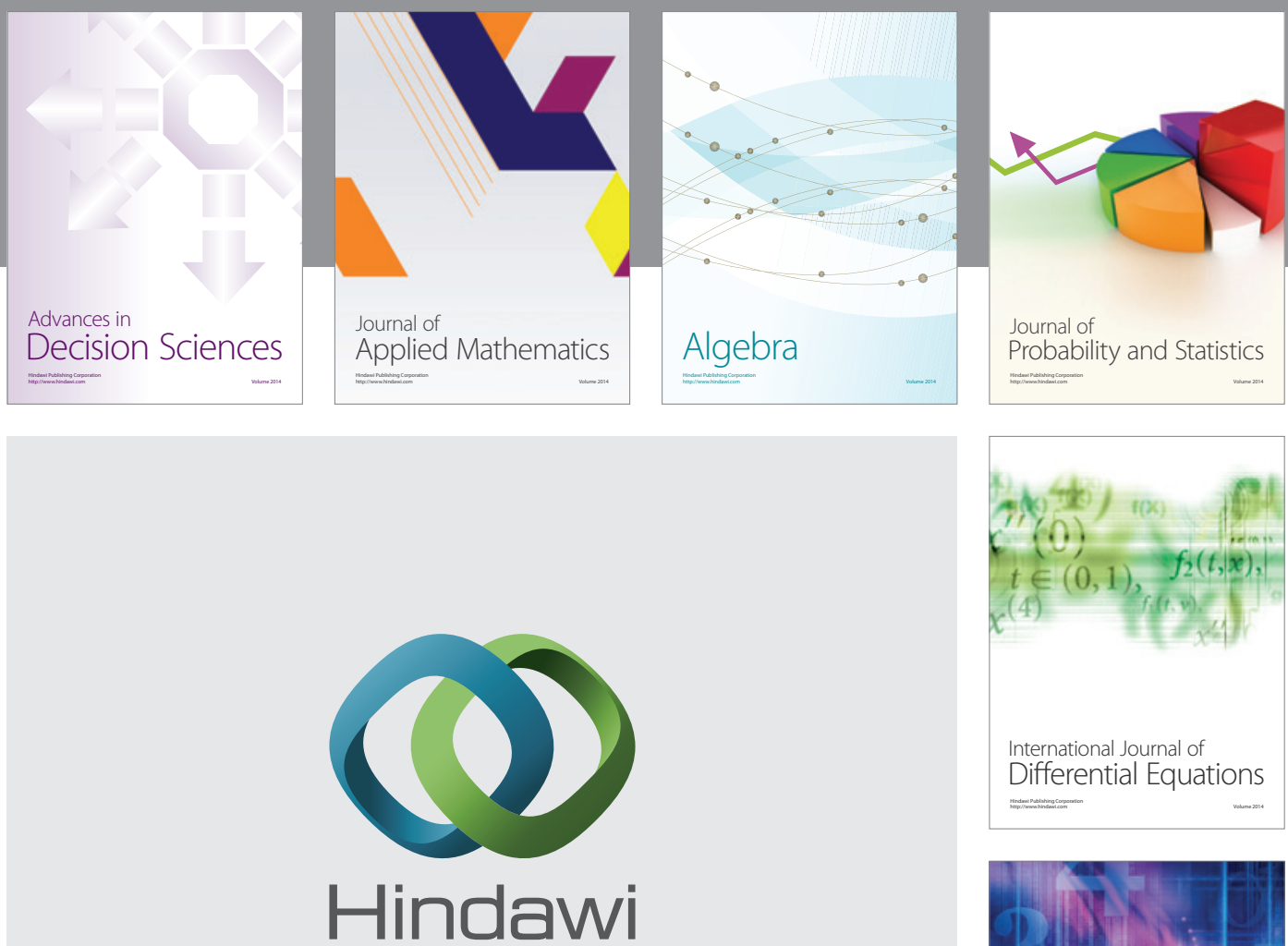

Submit your manuscripts at http://www.hindawi.com
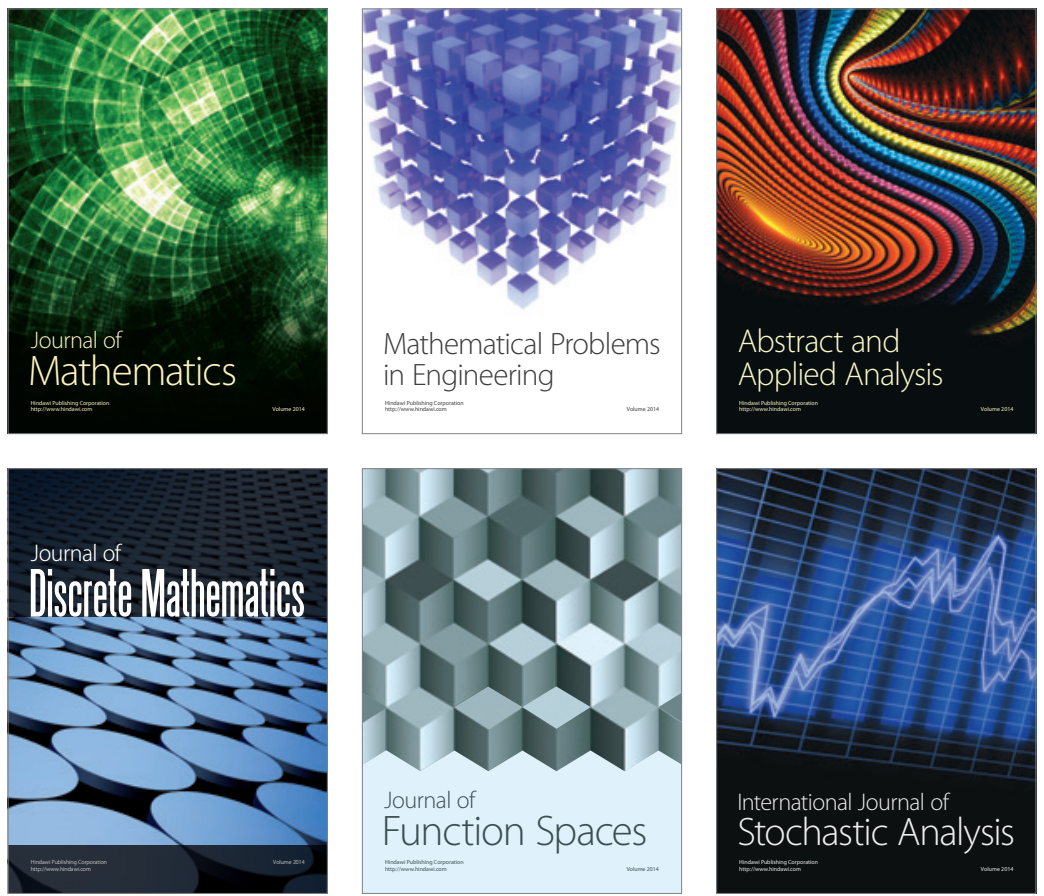

Journal of

Function Spaces

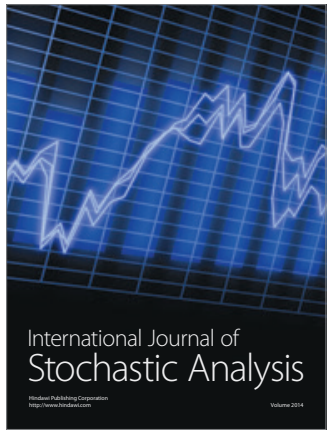

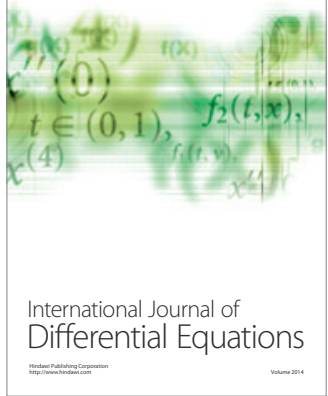
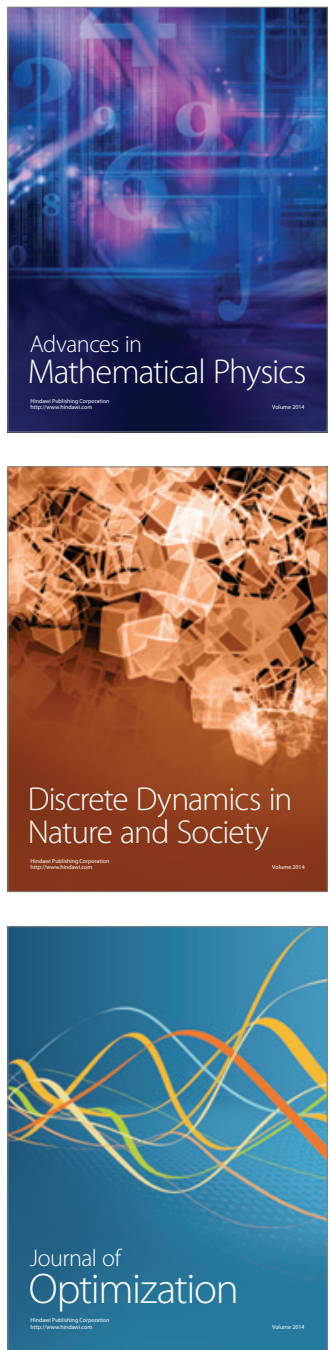